\title{
NO PERIODIC ORBITS IN THE BIANCHI MODELS B
}

\author{
JAUME LLIBRE* AND JIANG YU
}

\begin{abstract}
In this paper we prove that the Bianchi models B have no periodic solutions.
\end{abstract}

\section{IntRoduction AND STATEMENT OF THE MAIN RESUlts}

Bianchi $[1,2]$ was the first to classify three dimensional Lie algebras in terms of the reduction to the canonical forms for their structure constants. There are nine types of models based on the dimension $n$ of the algebra.

a) $n=0$ Type $I$;

b) $n=1$ Type $I I, I I I$;

c) $n=2$ Type $I V, V, V I, V I I$;

d) $n=3$ Type $V I I I, I X$.

To be of cosmological interest, this classification is called Bianchi universe models, which are spatially homogeneous cosmological models that in general are anisotropic. Taub [13] introduced this work of Bianchi into relativistic cosmology and derived the dynamic equations for the generic vacuum Bianchi geometries.

Ellis and MacCallum [8] gave this classification, introduced the type $\mathrm{A} / \mathrm{B}$ of Bianchi model notation that is now in common use [Type A: $a=0$, Type B: $a \neq 0$ ], see also [3]. The different types correspond to all nonequivalent sets of their structure constants. Let $\left\{X_{1}, X_{2}, X_{3}\right\}$ be an appropriate basis of the three dimensional Lie Algebra. Following Bogoyavlensky's approach, see [3], we can obtain the classification conditions on the property of the structure constants, and write in the form of commutation relations.

$$
\left[X_{1}, X_{2}\right]=n_{3} X_{3}, \quad\left[X_{2}, X_{3}\right]=n_{1} X_{1}-a X_{2}, \quad\left[X_{3}, X_{1}\right]=n_{2} X_{2}+a X_{1},
$$

where $a \in \mathbb{R},[$,$] is the Lie bracket and \left(n_{1}, n_{2}, n_{3}\right)$ with $n_{i} \in$ $\{+1,-1,0\}$. In particular, for $a=0$ we obtain models of type $A$ and

2010 Mathematics Subject Classification. 34C05, 34C25, 34C60.

Key words and phrases. Bianchi cosmological models, periodic orbits.

* This is the corresponding author. 
for $a \neq 0$ we obtain models of type $B$. we arrive at the following list of types $\mathrm{A}$ and $\mathrm{B}$ of homogeneous spaces. In the the table the roman numeral is labeled the type of the Bianchi classification

\begin{tabular}{|c|c|c|c|c|c|c|}
\hline Type & $I$ & $I I$ & $V I_{0}$ & $V I I_{0}$ & $V I I I$ & $I X$ \\
\hline$n_{1}$ & 0 & 1 & 1 & 1 & 1 & 1 \\
\hline$n_{2}$ & 0 & 0 & -1 & 1 & 1 & 1 \\
\hline$n_{3}$ & 0 & 0 & 0 & 0 & -1 & 1 \\
\hline
\end{tabular}

TABLE 1. The classification of Bianchi class A cosmologies.

\begin{tabular}{|c||c|c|c|c|c|}
\hline Type & $I I I$ & $I V$ & $V$ & $V I$ & $V I I$ \\
\hline$a$ & 1 & 1 & 1 & $a \neq 1$ & $a$ \\
\hline$n_{1}$ & 1 & 1 & 0 & 1 & 1 \\
\hline$n_{2}$ & -1 & 0 & 0 & -1 & 1 \\
\hline
\end{tabular}

TABLE 2. The classification of Bianchi class B cosmologies.

According to Bogoyavlensky [3], for the homogeneous cosmological models of Class B Einstein's system of equations reduces to the following dynamical system in the phase space $p_{i}, q_{i}, p_{\varphi}, \varphi, i=1,2,3$,

$$
\frac{d q_{i}}{d \tau}=\frac{\partial H}{\partial p_{i}}, \quad \frac{d p_{i}}{d \tau}=-\frac{\partial H}{\partial q_{i}}-h_{i}
$$

$$
\frac{d \varphi}{d \tau}=\frac{\partial H}{\partial p_{\varphi}}, \quad \frac{d p_{\varphi}}{d \tau}=-\frac{\partial H}{\partial \varphi}-h_{\varphi}
$$

where the function $H$ is

$$
H=\frac{1}{\left(q_{1} q_{2} q_{3}\right)^{\frac{1-k}{2}}}\left(T\left(p_{i} q_{i}\right)+V_{G}\left(q_{i}\right)\right)
$$

with

$$
\begin{aligned}
T\left(p_{i}, q_{i}, p_{\varphi}\right) & =2 \sum_{1 \leq i<j \leq 3} p_{i} p_{j} q_{i} q_{j}-\sum_{i=1}^{3} p_{i}^{2} q_{i}^{2}-\frac{p_{\varphi}^{2} q_{1} q_{2}}{\left(n_{1} q_{1}-n_{2} q_{2}\right)^{2}} \\
V_{G}\left(q_{i}\right) & =-\frac{1}{4}\left(12 a^{2} q_{1} q_{2}+\left(n_{1} q_{1}-n_{2} q_{2}\right)^{2}\right)
\end{aligned}
$$

and

$$
h_{1}=\frac{a^{2} q_{2}}{\left(q_{1} q_{2} q_{3}\right)^{\frac{1-k}{2}}}, \quad h_{2}=\frac{a^{2} q_{1}}{\left(q_{1} q_{2} q_{3}\right)^{\frac{1-k}{2}}},
$$




$$
h_{3}=\frac{-2 a^{2} q_{1} q_{2}}{q_{3}\left(q_{1} q_{2} q_{3}\right)^{\frac{1-k}{2}}}, \quad h_{\varphi}=\frac{a\left(n_{1} q_{1}-n_{2} q_{2}\right)^{2}}{\left(q_{1} q_{2} q_{3}\right)^{\frac{1-k}{2}}} .
$$

System (1) in an explicit form writes as

$$
\begin{aligned}
\frac{d q_{1}}{d \tau}= & -2 q_{1}\left(q_{1} q_{2} q_{3}\right)^{(k-1) / 2}\left(p_{1} q_{1}+p_{2} q_{2}-p_{3} q_{3}\right) \\
\frac{d q_{2}}{d \tau}= & -2 q_{2}\left(q_{1} q_{2} q_{3}\right)^{(k-1) / 2}\left(p_{1} q_{1}+p_{3} q_{3}-p_{2} q_{2}\right), \\
\frac{d q_{3}}{d \tau}= & -2 q_{3}\left(q_{1} q_{2} q_{3}\right)^{(k-1) / 2}\left(p_{1} q_{1}+p_{2} q_{2}-p_{3} q_{3}\right), \\
\frac{d p_{1}}{d \tau}= & -\left(q_{1} q_{2} q_{3}\right)^{(k-1) / 2}\left(2 p_{1}\left(p_{2} q_{2}+p_{3} q_{3}-p_{1} q_{1}\right)\right. \\
+ & \left.\frac{p_{\varphi}^{2}\left(n_{1} q_{1}+n_{2} q_{2}\right)}{\left(n_{1} q_{1}-n_{2} q_{2}\right)^{3}} q_{2}-\frac{1}{2} n_{1}\left(n_{1} q_{1}-n_{2} q_{2}\right)-2 a^{2} q_{2}\right)+\frac{1-k}{2 q_{1}} \bar{H} \\
\frac{d p_{2}}{d \tau}= & -\left(q_{1} q_{2} q_{3}\right)^{(k-1) / 2}\left(2 p_{2}\left(p_{1} q_{1}+p_{3} q_{3}-p_{2} q_{2}\right)\right. \\
& \left.-\frac{p_{\varphi}^{2}\left(n_{1} q_{1}+n_{2} q_{2}\right)}{\left(n_{1} q_{1}-n_{2} q_{2}\right)^{3}} q_{1}-\frac{1}{2} n_{2}\left(n_{1} q_{1}-n_{2} q_{2}\right)-2 a^{2} q_{1}\right)+\frac{1-k}{2 q_{2}} \bar{H} \\
\frac{d p_{3}}{d \tau}= & -\left(q_{1} q_{2} q_{3}\right)^{(k-1) / 2}\left(2 p_{3}\left(p_{1} q_{1}+p_{2} q_{2}-p_{3} q_{3}\right)-2 a^{2} \frac{q_{1} q_{2}}{q_{3}}\right) \\
& +\frac{1-k}{2 q_{3}} \bar{H}, \\
\frac{d \varphi}{d \tau}= & -\left(q_{1} q_{2} q_{3}\right)^{(k-1) / 2} \frac{2 q_{1} q_{2} p_{\varphi}}{\left(n_{1} q_{1}-n_{2} q_{2}\right)^{2}}, \\
\frac{d p_{\varphi}}{d \tau}= & -\left(q_{1} q_{2} q_{3}\right)^{(k-1) / 2} a\left(n_{1} q_{1}-n_{2} q_{2}\right)^{2}
\end{aligned}
$$

with $0 \leq k \leq 1$ and $\bar{H}=T+V_{G}$.

Using dynamical systems methods, Collins [7] first introduce phase planes with compactified boundaries, to characterize the evolution of particular Bianchi classes of universe models, and Bogoyavlensky systematically introduce its study and application in [3]. After that many dynamical properties of the Bianchi models have been studied, for example, the integrability and the existence of periodic orbits, see $[4,5,6,9,10,12]$ and the references quoted there.

In this paper we study the periodic solutions of the type B of Bianchi model (2). It is known that all the Bianchi class A models do not have periodic orbits. Using evolutions equations associated to these models, and showing that such equations always have some monotone function evaluated on the orbits, Wainwright and Ellis [14] prove that these models cannot exhibit periodic motion. Buzzi and Llibre [4] provide a new, direct and easier proof on the non-existence of periodic orbits for the 6 models of Bianchi class A. On the other hand for the Einstein's field equation, Llibre and Yu [11] 
studied the periodic orbits of the static, spherically symmetric EinsteinYang-Mills equations. We shall mainly follow the idea of the qualitative analysis of dynamical systems shown in [4] and [11] in this paper.

Our main result is the following.

Theorem 1. The Bianchi models B, i.e. III, IV, V, VI and VII in Table 2 have no periodic solutions.

We shall provide the proof of Theorem 1 for the type $\mathrm{V}$ of the Bianchi models B in section 2, the proof of Theorem 1 for the type IV of the Bianchi models B in section 3, and the proof of Theorem 1 for the type III, VI and VII of the Bianchi models B in section 4.

\section{The Bianchi V MOdel}

Consider Bianchi V model. Noticing that for Bianchi $\mathrm{V}$ we have $p_{\varphi}=0$ and $\dot{\varphi}=0$. After the change of coordinates and time

$$
x_{i}=q_{i}, x_{i+3}=p_{i} q_{i}, i=1,2,3, d \tau_{0}=\frac{\left(q_{1} q_{2} q_{3}\right)^{\frac{k-1}{2}}}{2} d \tau,
$$

we obtain a simplest model of six equations, and write it as the homogeneous polynomial differential system of degree 2 .

$$
\begin{aligned}
& \dot{x}_{1}=x_{1}\left(-x_{4}+x_{5}+x_{6}\right), \\
& \dot{x}_{2}=x_{2}\left(x_{4}-x_{5}+x_{6}\right), \\
& \dot{x}_{3}=x_{3}\left(x_{4}+x_{5}-x_{6}\right), \\
& \dot{x}_{4}=x_{1} x_{2}+\frac{k-1}{4}\left(3 x_{1} x_{2}+\Lambda\right), \\
& \dot{x}_{5}=x_{1} x_{2}+\frac{k-1}{4}\left(3 x_{1} x_{2}+\Lambda\right), \\
& \dot{x}_{6}=x_{1} x_{2}+\frac{k-1}{4}\left(3 x_{1} x_{2}+\Lambda\right),
\end{aligned}
$$

where $0 \leq k \leq 1$ and

$$
\Lambda=x_{4}^{2}+x_{5}^{2}+x_{6}^{2}-2\left(x_{4} x_{5}+x_{4} x_{6}+x_{5} x_{6}\right) .
$$

Proposition 2. The Bianchi $V$ system (3) has no periodic solutions.

Proof. Suppose that system (3) has a periodic solution $\Gamma(t)=\left(x_{1}, x_{2}\right.$, $\left.x_{3}, x_{4}, x_{5}, x_{6}\right)(t)$ with period $T>0$. We have from (3) that $\dot{x}_{4}=\dot{x}_{5}=\dot{x}_{6}$, then

$$
x_{4}=x_{6}+a \quad \text { and } \quad x_{5}=x_{6}+b,
$$

where $a$ and $b$ are any constants in $\mathbb{R}$. Substituting into the first three equations of (3) we have

$$
\begin{aligned}
& \dot{x}_{1}=x_{1}\left(b-a+x_{6}\right), \\
& \dot{x}_{2}=x_{2}\left(a-b+x_{6}\right), \\
& \dot{x}_{3}=x_{3}\left(a+b-x_{6}\right) .
\end{aligned}
$$


From this differential system it follows that

$$
\frac{d\left(x_{1} x_{3}\right)}{d t}=2 b\left(x_{1} x_{3}\right), \frac{d\left(x_{2} x_{3}\right)}{d t}=2 a\left(x_{2} x_{3}\right) .
$$

Hence we get $x_{1} x_{3}=C_{1} e^{2 b t}$ and $x_{2} x_{3}=C_{2} e^{2 a t}$. Since the exponential function is not periodic, so the Bianchi V system (3) has no periodic solutions.

\section{The Bianchi IV MODEL}

Consider Bianchi IV model, after the change of coordinates and time

$$
x_{i}=q_{i}, x_{i+3}=p_{i} q_{i}, i=1,2,3, d \tau_{0}=\frac{q_{1}\left(q_{1} q_{2} q_{3}\right)^{\frac{k-1}{2}}}{2} d \tau,
$$

system (2) can be written as the six-dimensional homogeneous polynomial differential system of degree 3 .

$$
\begin{aligned}
& \dot{x}_{1}=x_{1}^{2}\left(-x_{4}+x_{5}+x_{6}\right), \\
& \dot{x}_{2}=x_{1} x_{2}\left(x_{4}-x_{5}+x_{6}\right), \\
& \dot{x}_{3}=x_{1} x_{3}\left(x_{4}+x_{5}-x_{6}\right), \\
& \dot{x}_{4}=\frac{x_{1}^{3}}{4}+x_{1}^{2} x_{2}-2 x_{2}\left(x_{4}-x_{5}+x_{6}\right)^{2}+\frac{k-1}{4} R, \\
& \dot{x}_{5}=x_{1}^{2} x_{2}+2 x_{2}\left(x_{4}-x_{5}+x_{6}\right)^{2}+\frac{k-1}{4} R, \\
& \dot{x}_{6}=x_{1}^{2} x_{2}+\frac{k-1}{4} R,
\end{aligned}
$$

where $0 \leq k \leq 1$ and

$$
R=\frac{x_{1}^{3}}{4}+3 x_{1}^{2} x_{2}+4 x_{2}\left(x_{4}+x_{5}-2 x_{6}\right)^{2}+x_{1} \Lambda .
$$

Lemma 3. System (4) has an invariant hyperplane $\left\{x_{1}=0\right\}$ and no periodic solution in this hyperplane.

Proof. Obviously the hyperplane $\left\{x_{1}=0\right\}$ is invariant manifold for system (4).

Suppose that system (4) has a periodic solution $\Gamma(t)=\left(x_{1}, x_{2}, x_{3}, x_{4}\right.$, $\left.x_{5}, x_{6}\right)(t)$ with period $T>0$ in $x_{1}=0$. We have that $x_{1}=0, x_{2}=a$ and $x_{3}=b$ where $a, b$ are constants, and $x_{4}, x_{5}, x_{6}$ satisfy

$$
\begin{aligned}
& \dot{x}_{4}=a(k-3)\left(x_{4}-x_{5}+x_{6}\right)^{2}, \\
& \dot{x}_{5}=a(k+1)\left(x_{4}-x_{5}+x_{6}\right)^{2}, \\
& \dot{x}_{6}=a(k-1)\left(x_{4}-x_{5}+x_{6}\right)^{2} .
\end{aligned}
$$

The set of singular points of (5) is the plane $x_{4}-x_{5}+x_{6}=0$. All the orbits of (5) shall be attracted or repelled by this plane. Moreover $x_{4}, x_{5}$ and $x_{6}$ are monotone. Hence if $\left(x_{4}, x_{5}, x_{6}\right)$ is a periodic solution of $(4)$, then $\left(x_{4}, x_{5}, x_{6}\right)$ has to be a singular point in the phase portrait of (5), i.e. a constant vector. So $\Gamma(t)$ is a singular point instead of a periodic solution. 
Proposition 4. Bianchi IV system (4) has no periodic solutions.

Proof. In view of Lemma 3 we consider that system (4) has a periodic solution $\Gamma(t)=\left(x_{1}, x_{2}, x_{3}, x_{4}, x_{5}, x_{6}\right)(t)$ with period $T>0$ contained in the region $x_{1} \neq 0$. From (4) we have $x_{4}^{\prime}+x_{5}^{\prime}-2 x_{6}^{\prime}=x_{1}^{3} / 4 \neq 0$. If the function $x_{4}(t)+x_{5}(t)-2 x_{6}(t)$ is a periodic function, then there is some $t_{0}$ such that its derivative is zero, so $x_{1}^{3}\left(t_{0}\right)=4\left(x_{4}^{\prime}\left(t_{0}\right)+x_{5}^{\prime}\left(t_{0}\right)-2 x_{6}^{\prime}\left(t_{0}\right)\right)=0$, which is a contradiction. So we finish the proof of Proposition 4.

\section{The Bianchi III, VI AND VII MOdel}

Consider the rest of the cases, i.e. the type III, VI and VIII of Bianchi models. After the change of coordinates and time

$$
\begin{aligned}
x_{i} & =q_{i}, x_{i+3}=p_{i} q_{i}, \quad i=1,2,3, \\
\frac{d \tau_{0}}{d \tau} & =\frac{N^{3}\left(q_{1} q_{2} q_{3}\right)^{\frac{k-1}{2}}}{2},
\end{aligned}
$$

where $N=x_{1}-n_{2} x_{2}$ and $n_{2}= \pm 1$, system (2) can be written as the six-dimensional homogeneous polynomial differential system of degree 5 .

$$
\begin{aligned}
\dot{x}_{1}= & x_{1} N^{3}\left(-x_{4}+x_{5}+x_{6}\right), \\
\dot{x}_{2}= & x_{2} N^{3}\left(x_{4}-x_{5}+x_{6}\right), \\
\dot{x}_{3}= & x_{3} N^{3}\left(x_{4}+x_{5}-x_{6}\right), \\
\dot{x}_{4}= & \frac{1}{4} x_{1} N^{4}+a^{2} x_{1} x_{2} N^{3}+\frac{k-1}{4} N S \\
& -2 a^{2} x_{1} x_{2}\left(x_{1}+n_{2} x_{2}\right)\left(x_{4}-x_{5}+x_{6}\right)^{2}, \\
\dot{x}_{5}= & \frac{1}{4} N^{5}-\frac{1}{4} x_{1} N^{4}+a^{2} x_{1} x_{2} N^{3}+\frac{k-1}{4} N S \\
& +2 a^{2} x_{1} x_{2}\left(x_{1}+n_{2} x_{2}\right)\left(x_{4}-x_{5}+x_{6}\right)^{2}, \\
\dot{x}_{6}= & a^{2} x_{1} x_{2} N^{3}+\frac{k-1}{4} N S,
\end{aligned}
$$

where $0 \leq k \leq 1$ and

$$
S=N^{4} / 4+4 a^{2} x_{1} x_{2}\left(x_{1}+n_{2} x_{2}\right)\left(x_{4}+x_{5}-2 x_{6}\right)^{2}+N^{2}\left(3 a^{2} x_{1} x_{2}+\Lambda\right) .
$$

Lemma 5. System (6) has an invariant hyperplane $\{N=0\}$ and no periodic solution in this hyperplane.

Proof. Let $\mathcal{X}$ the vector field associated to the differential system (6). Hence

$$
\mathcal{X}(N)=\dot{x}_{1}-n_{2} \dot{x}_{2}=N^{3}\left(\left(x_{1}+n_{2} x_{2}\right)\left(-x_{4}+x_{5}\right)+N x_{6}\right) .
$$

The hyperplane $N=0$ is invariant by the flows of system (6).

Suppose that system (6) has a periodic solution $\Gamma(t)=\left(x_{1}, x_{2}, x_{3}, x_{4}\right.$, $\left.x_{5}, x_{6}\right)(t)$ with period $T>0$ in $N=0$. Then we have that $x_{1}=c_{1}, x_{2}=c_{2}$ and $x_{3}=c_{3}$ where $c_{i}$ are constants, and $x_{4}, x_{5}, x_{6}$ satisfy

$$
\begin{aligned}
& \dot{x}_{4}=-2 a^{2} c_{1} c_{2}\left(c_{1}+n_{2} c_{2}\right)\left(x_{4}-x_{5}+x_{6}\right)^{2}, \\
& \dot{x}_{5}=2 a^{2} c_{1} c_{2}\left(c_{1}+n_{2} c_{2}\right)\left(x_{4}-x_{5}+x_{6}\right)^{2}, \\
& \dot{x}_{6}=0 .
\end{aligned}
$$


Hence if $\left(x_{4}, x_{5}, x_{6}\right)$ is a periodic solution of (7), using the same kind of arguments as in the proof of Lemma $3,\left(x_{4}, x_{5}, x_{6}\right)$ has to be a constant vector, i.e. a singular point in the phase portrait of $(7)$. So $\Gamma(t)$ is a singular point instead of a periodic solution.

Proposition 6. The type III, VI and VII of Bianchi system (6) has no periodic solutions.

Proof. In view of Lemma 5, we consider that system (6) has a periodic solution $\Gamma(t)=\left(x_{1}, x_{2}, x_{3}, x_{4}, x_{5}, x_{6}\right)(t)$ with period $T>0$ contained in the region $N \neq 0$. By the time rescaling $d \tau=N d t$, system (6) can be written as

$$
\begin{aligned}
x_{1}^{\prime}= & x_{1} N^{2}\left(-x_{4}+x_{5}+x_{6}\right), \\
x_{2}^{\prime}= & x_{2} N^{2}\left(x_{4}-x_{5}+x_{6}\right), \\
x_{3}^{\prime}= & x_{3} N^{2}\left(x_{4}+x_{5}-x_{6}\right), \\
x_{4}^{\prime}= & \frac{1}{4} x_{1} N^{3}+a^{2} x_{1} x_{2} N^{2}+\frac{k-1}{4} S \\
& -2 a^{2} x_{1} x_{2}\left(x_{1}+n_{2} x_{2}\right)\left(x_{4}-x_{5}+x_{6}\right)^{2} / N, \\
x_{5}^{\prime}= & \frac{1}{4} N^{4}-\frac{1}{4} x_{1} N^{3}+a^{2} x_{1} x_{2} N^{2}+\frac{k-1}{4} S \\
& +2 a^{2} x_{1} x_{2}\left(x_{1}+n_{2} x_{2}\right)\left(x_{4}-x_{5}+x_{6}\right)^{2} / N, \\
x_{6}^{\prime}= & a^{2} x_{1} x_{2} N^{2}+\frac{k-1}{4} S,
\end{aligned}
$$

where $^{\prime}=d / d \tau$. From (8) we have $x_{4}^{\prime}(\tau)+x_{5}^{\prime}(\tau)-2 x_{6}^{\prime}(\tau)=N^{4} / 4 \neq 0$. If $\Gamma(t)$ is a periodic orbit of (6) in time variable $t$, then it is also a periodic orbit of (8) in time variable $\tau$. Then the periodic function $x_{4}(t)+x_{5}(t)-$ $2 x_{6}(t)$ in the variable $t$ implies that $x_{4}(\tau)+x_{5}(\tau)-2 x_{6}(\tau)$ is also a periodic function in $\tau$. Hence there is some $\tau_{0}$ such that the derivative of the function $x_{4}(\tau)+x_{5}(\tau)-2 x_{6}(\tau)$ is zero, i.e. $N^{4}\left(\tau_{0}\right)=4\left(x_{4}^{\prime}\left(\tau_{0}\right)+x_{5}^{\prime}\left(\tau_{0}\right)-2 x_{6}^{\prime}\left(\tau_{0}\right)\right)=0$, which is a contradiction. This completes the proof of Proposition 6.

Proof of Theorem 1. From Propositions 2, 4 and 6, Theorem 1 follows.

Acknowledgements. The first author is partially supported by the MINECO grants MTM2016-77278-P and MTM2013-40998-P, an AGAUR grant number 2014SGR-568, and the grants FP7-PEOPLE-2012-IRSES 318999. and from the recruitment program of high-end foreign experts of China.

The second author is partially supported by the state key program of NNSF of China grant number 11431008, NSF of Shanghai grant number 15ZR1423700.

\section{REFERENCES}

[1] L. BIANCHI, Sugli spazi a tre dimensioni che ammettono un gruppo continuo di movimenti (On the spaces of three dimensions that admit a continuous group of movements), Soc. Ital. Sci. Mem. di Mat. 11 (1898), 267-352. 
[2] L. BIANCHI, Lezioni sulla teoria dei gruppi continui finiti di transformazioni (Lectures on the theory of finite continuous transformation groups), Spoerri, Pisa (1918), 550-557.

[3] O.I. Bogoyavlensky, Methods in the qualitative theory of dynamical systems in astrophysics and gas dynamics, Springer Series in Soviet Mathematics, Springer-Verlag, Berlin, 1985.

[4] C.A. Buzzi And J. Llibre, No periodic orbits for the type A Bianchi's systems, J. Nonlinear Math. Phys. 22(2015), 170-179.

[5] C.A. Buzzi, J. Llibre And P.R. DA Silva, On the dynamics of the Bianchi IX System, J. Phys. A: Math. Gen. 40 (2007), 7187-7192.

[6] J. Chavarriga And I.A. García, Integrability and explicit solutions in some Bianchi cosmological dynamical systems, J. Nonlinear Math. Phys. 8 (2001), 96-105.

[7] C.B. Collins, More qualitative cosmology, Comm. Math. Phys. 23(1971), $137-158$.

[8] G. Ellis and M. MacCallum, A class of homogeneous cosmological models, Comm. Math. Phys. 12(1969), 108-141.

[9] A. Ferragut, J. Llibre And C. Pantazi, Analytic Integrability of Bianchi A cosmological models, J. of Geometry and Physics 62 (2012), 381-386.

[10] R.M. GAD AND A.S. AlOFI, Homothetic vector field in plane symmetric Bianchi type-I cosmological model in Lyra geometry, Modern Phys. Lett. A 29 (2014), no. 22, 1450116, 12 pp.

[11] J. LlibRe AND J. YU, On the periodic orbits of the static, spherically symmetric Einstein-Yang-Mills equations, Comm. Math. Phys. 286(2009), 277-281.

[12] K.E. Starkov, Compact invariant sets of the Bianchi VIII and Bianchi IX Hamiltonian systems, Phys. Lett. A 375 (2011), no. 36, 3184-3187.

[13] A. H. TAuB, Empty space-times admitting a three parameter group of motions, Ann. Math. 53 (1951), 472-490.

[14] J. Wainwright And G.F.R. Ellis, Dynamical systems in cosmology, Cambridge University Press, Cambridge, 1997.

JAUME LLIBRE

Departament de Matemàtiques, Universitat Autònoma de Barcelona, 08193 Bellaterra, Barcelona, Catalonia, Spain

E-mail address: jllibre@mat.uab.cat

JIANG YU

Department of Mathematics, Shanghai Jiao Tong University, Shanghai 200240, CHINA

E-mail address: jiangyu@sjtu.edu.cn 